\title{
EXCEPTIONAL SCATTEREDNESS IN PRIME DEGREE
}

\author{
ANDREA FERRAGUTI AND GIACOMO MICHELI
}

\begin{abstract}
Let $q$ be an odd prime power and $n$ be a positive integer. Let $\ell \in \mathbb{F}_{q^{n}}[x]$ be a $q$-linearised $t$-scattered polynomial of linearized degree $r$. Let $d=\max \{t, r\}$ be an odd prime number. In this paper we show that under these assumptions it follows that $\ell=x$. Our technique involves a Galois theoretical characterization of $t$-scattered polynomials combined with the classification of transitive subgroups of the general linear group over the finite field $\mathbb{F}_{q}$.
\end{abstract}

\section{INTRODUCTION}

Scattered polynomials have risen a great deal of interest recently, due to their connections to Desarguesian spreads and maximal rank distance codes, which are used in the context of network coding. In fact, from a scattered polynomial one can construct scattered sets with respect to the Desarguesian spread (which are interesting objects in their own arising in finite geometry $[5,14]$ ) and in turn a maximal rank distance code for some parameters (see $[15,16]$ ). Scatteredness is an extremely rare condition for a polynomial [2, Section 1]; this suggests the possibility of classifying all exceptional $t$-scattered polynomials (see $[1,2]$ ). We recall below the definition of such objects. For a linearized polynomial $\sum_{i=0}^{r} a_{i} x^{q^{i}}$ with $a_{r} \neq 0$, the linearized degree is the non-negative integer $r$.

Definition 1.1. Let $q$ be a prime power, $n, m$ be positive integers, $t$ be a non-negative integer and $\ell$ be a $q$-linearised polynomial in $\mathbb{F}_{q^{n}}[x]$ of linearised degree $r$. Then $\ell$ is said to be $(q, n, m, t)$-scattered if, for any $s_{0} \in \mathbb{F}_{q^{n m}}$, the polynomial $f-s_{0} x^{q^{t}}$ has at most $q$ roots. The non-negative integer $t$ is called index of $\ell$. If, fixed $q, n$ and $t$, the polynomial $\ell$ is $(q, n, m, t)$-scattered for infinitely many $m$ 's, we say that $\ell$ is exceptional $t$-scattered.

For $t=0$ exceptional $t$-scattered polynomials coincide with exceptional scattered polynomials, i.e. polynomials that are scattered for infinitely many extensions of the base field. On the other hand, if one finds a polynomial $\ell$ that is exceptional $t$-scattered, for

2010 Mathematics Subject Classification. 11 T06.

Key words and phrases. Scattered Polynomials, Exceptionality, Rank Metric Codes, Scattered Linear Sets, Chebotarev Density Theorem; Finite Fields; Galois Theory. 
$t>0$, then one automatically finds infinitely many scattered polynomials (over different fields) as follows: for any $m$ such that $\ell$ is $t$-scattered over $\mathbb{F}_{q^{n m}}$, consider the polynomial $\ell\left(x^{q^{n m-t}}\right)$. It is elementary to see $[2$, Section 1$]$ that $\ell\left(x^{q^{n m-t}}\right)$ is scattered over $\mathbb{F}_{q^{n m}}$.

In this paper we consider the problem of classifying exceptional $t$-scattered polynomials. This question has already been studied in the literature, leading to classification results in the case $t=0$ and $t=1[1,2]$. Therefore, in the rest of the paper we simply restrict to the case $t \geq 1$, which simplifies some of the proofs without impacting on the generality of the results. Our work contains two main results: first we provide a Galois theoretical characterization of exceptional $t$-scattered polynomials (Theorem 2.6); subsequently we use the latter to prove the following classification result.

Theorem 1.2. Let $q$ be an odd prime power and let $t \geq 1$. Let $\ell$ be an exceptional $t$ normalized $t$-scattered polynomial of linearized degree $r$, and let $d:=\max \{r, t\}$. Suppose that $d$ is an odd prime. Then $\ell=x$.

The strategy of our proof is to exploit the full power of the classification of transitive subgroups of the general linear group [6,9] via our Galois theoretical characterization. It is worth noticing that our approach describes a new, completely equivalent condition to exceptional scatteredness.

In order to classify exceptional scattered polynomials, it is enough to consider those in a canonical form, which we are about to recall.

Remark 1.3. As noticed in $[2$, p. 511], if $\ell$ is $(q, n, m, t)$-scattered we can make the following assumptions without loss of generality:

- $\ell$ is monic;

- the coefficient of the term $x^{q^{t}}$ of $\ell$ is zero;

- if $t>0$ then the linear term of $\ell$ is non-zero.

In particular, notice that we can always assume that $t$ differs from the linear degree of $\ell$.

Definition 1.4. A linearized polynomial satisfying the properties of Remark 1.3 will be called t-normalized.

\section{A Galois theoretical Characterization of $t$-Normalized exceptional} SCATTERED POLYNOMIALS

In this section we provide a Galois theoretical characterization of $t$-normalized exceptional scattered polynomials of positive index. We will use the notation and the 
terminology of [17]. We start by briefly recalling the setup and two auxiliary lemmata. Let $K$ be a function field with constant field $\mathbb{F}_{q}$ and let $M$ be a Galois extension of $K$ with constant field $k$ (the field $k$ is a finite extension of $\mathbb{F}_{q}$ ). We denote by $G^{\text {arith }}:=\operatorname{Gal}(M / K)$ the arithmetic Galois group of the extension $M / K$. For every place $P$ of $K$ and every place $R$ of $M$ lying above $P$, the decomposition group $D(R / P) \subseteq G^{\text {arith }}$ is the set of all automorphisms fixing $R$ as a set. If $k_{P}$ and $k_{R}$ are the residue fields at $P$ and $R$, respectively, there is a surjective map $\pi_{R}: D(R \mid P) \rightarrow \operatorname{Gal}\left(k_{R} / k_{P}\right)$ whose kernel, denoted by $I(R \mid P)$, is called inertia group. A Frobenius for $R \mid P$ is any preimage, via $\pi_{R}$, of the Frobenius automorphism $u \mapsto u^{q}$ of the extension of finite fields $k_{R} / k_{P}$. The geometric Galois group of the extension $M / K$ is defined as $G^{\text {geom }}:=\operatorname{Gal}(M / k \cdot K)$. This is a normal subgroup of $G^{\text {arith }}$, and there is an isomorphism $\varphi: G^{\text {arith }} / G^{\text {geom }} \rightarrow \operatorname{Gal}\left(k / \mathbb{F}_{q}\right)$. Finally, for any $m \geq 1$ we denote by $M_{m}$ and $K_{m}$ the composita $M \cdot \mathbb{F}_{q^{m}}$ and $K \cdot \mathbb{F}_{q^{m}}$, respectively. The corresponding arithmetic and geometric Galois groups will be denoted by $G_{m}^{\text {arith }}$ and $G_{m}^{\text {geom }}$, the field of constants of $M_{m}$ will be denoted by $k_{m}$, and $\varphi_{m}$ will be the isomorphism $G_{m}^{\text {arith }} / G_{m}^{\text {geom }} \rightarrow \operatorname{Gal}\left(k_{m} / \mathbb{F}_{q^{m}}\right)$. The reader should notice that the isomorphism class of $G_{m}^{\text {geom }}$ is independent of $m$, while $G_{m}^{\text {arith }}$ might differ from $G^{\text {arith }}$.

Lemma 2.1. Let $M / K$ be a Galois extension of global function fields. Assume that the constant field of $K$ is $\mathbb{F}_{q}$ and let $k$ be the constant field of $M$. Then there exists a constant $C \in \mathbb{R}^{+}$, depending only on the degree of the extension $M / K$, with the following property: if $q^{m}>C$ then every $\gamma \in G_{m}^{\text {arith }}$ such that $\varphi_{m}(\gamma)$ is the Frobenius automorphism for the extension $k_{m} / \mathbb{F}_{q^{m}}$ is also the Frobenius of a finite, unramified place of degree 1 of $K_{m}$.

Proof. This is an immediate consequence of Chebotarev density theorem for function fields, see for example [7, Remark 2.3] or [11].

Definition 2.2. Let $M / K$ be a Galois extension of function fields. We call the smallest constant $C$ such that the conclusion of Lemma 2.1 holds a uniformizing constant for $M / K$.

In other words, $C$ is the smallest size of a finite field $\mathbb{F}_{q^{m_{0}}}$ such that for every $m \geq m_{0}$, every element of $G_{m}^{\text {arith }}$ that lies in the coset $G_{m}^{\text {geom }} \gamma$ is the Frobenius of a finite, unramified place of $K$.

The following lemma is a classical fact from algebraic number theory, whose proof can be found for example in [8].

Lemma 2.3. Let $L / K$ be a finite separable extension of global function fields, let $M$ be its Galois closure and $G:=\operatorname{Gal}(M / K)$ be its (arithmetic) Galois group. Let $P$ be a place 
of $K$ and $\mathcal{Q}$ be the set of places of $L$ lying above $P$. Let $R$ be a place of $M$ lying above $P$. The following hold:

(1) There is a natural bijection between $\mathcal{Q}$ and the set of orbits of $H:=\operatorname{Hom}_{K}(L, M)$ under the action of the decomposition group $D(R \mid P)=\{g \in G \mid g(R)=R\}$.

(2) Let $Q \in \mathcal{Q}$ and let $H_{Q}$ be the orbit of $D(R \mid P)$ corresponding to $Q$. Then $\left|H_{Q}\right|=e(Q \mid P) f(Q \mid P)$ where $e(Q \mid P)$ and $f(Q \mid P)$ are ramification index and relative degree, respectively.

(3) The orbit $H_{Q}$ partitions further under the action of the inertia group $I(R \mid P)$ into $f(Q \mid P)$ orbits of size $e(Q \mid P)$.

The above lemmata allows to convert splitting conditions of intermediate extension into group theoretical ones (see $[12,13]$ for more applications of these lemmata not related to exceptionality ).

A function field theoretical setup for scattered polynomials. Let $\ell \in \mathbb{F}_{q^{n}}[x]$ be a $q$-linearized polynomial of linearized degree $r \geq 0$ and such that the linear term of $\ell$ is non-zero. Let $s$ be transcendental over $\mathbb{F}_{q}$ and let $t>0$. We will call $d:=\max \{r, t\}$ the $t$-scatter-degree of $f$. Let $M$ be the splitting field of $\ell-s x^{q^{t}}$ over $\mathbb{F}_{q^{n}}(s)$. For every $m \geq 1$, we denote by $M_{m}$ the compositum $M \cdot \mathbb{F}_{q^{n m}}$ and by $G_{m}^{\text {arith }}:=\operatorname{Gal}\left(M_{m} / \mathbb{F}_{q^{n m}}(s)\right)$ the arithmetic Galois group of the extension $M_{m} / \mathbb{F}_{q^{n m}}(s)$. The field of constants of such extension, which is defined as $\overline{\mathbb{F}}_{q} \cap M_{m}$, will be denoted by $k_{m}$. We denote by $G_{m}^{\text {geom }}:=\operatorname{Gal}\left(M_{m} / k_{m} \cdot \mathbb{F}_{q}(s)\right)$ the geometric Galois group. We will denote by $\varphi_{m}$ the isomorphism $G_{m}^{\text {arith }} / G_{m}^{\text {geom }} \rightarrow \operatorname{Gal}\left(k_{m} / \mathbb{F}_{q^{n m}}\right)$. Finally, we will denote by $V$ the set of roots of $\ell-s x^{q^{t}}$ in an algebraic closure $\overline{\mathbb{F}_{q}(s)}$ of $\mathbb{F}_{q}(s)$.

One can immediately deduce the following corollary from Lemma 2.3.

Corollary 2.4. Let $t \geq 1$ and let $\ell$ be a linearized polynomial in $t$-normalized form. Let $\alpha$ be a root of $\ell / x-s x^{q^{t}-1}$ and let $L:=\mathbb{F}_{q^{n m}}(\alpha)$. Let $P$ be a place of $\mathbb{F}_{q^{n m}}(s)$ and $\mathcal{Q}$ be the set of places of $L$ lying above $P$. Let $R$ be a place of $M_{m}$ lying above $P$. Then the following hold:

(1) There is a natural bijection between $\mathcal{Q}$ and the set of orbits of $V$ under the action of the decomposition group $D(R \mid P)$.

(2) Let $Q \in \mathcal{Q}$ and let $V_{Q}$ be the orbit of $D(R \mid P)$ corresponding to $Q$. Then $\left|V_{Q}\right|=$ $e(Q \mid P) f(Q \mid P)$ where $e(Q \mid P)$ and $f(Q \mid P)$ are the ramification index and the relative degree, respectively. 
Proof. Using Lemma 2.3, it is enough to observe that since $\ell$ is $t$-normalized, then the polynomial $\ell / x-s x^{q^{t}-1}$ is separable and it is irreducible because $\operatorname{gcd}\left(\ell / x, x^{q^{t}-1}\right)=1$. Hence, $\operatorname{Hom}_{K}(L, M)$ and the set $V \backslash\{0\}$ of roots of $\ell / x-s x^{q^{t}-1}$ are isomorphic $D(R \mid P)$ sets.

With this notation, we are now ready to state and prove the Galois theoretical characterization of exceptional scattered polynomials, which will allow to encode scatteredness in group theoretical terms.

Remark 2.5. Notice that the set $V$ of roots of $\ell-s x^{q^{t}}$ is an $\mathbb{F}_{q^{-}}$vector space. Since $G^{\text {arith }}$

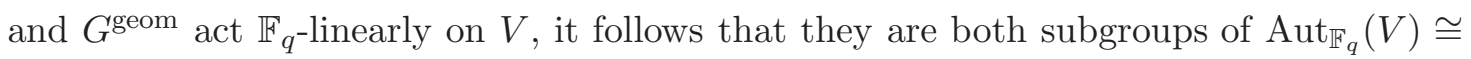
$\mathrm{GL}_{d}\left(\mathbb{F}_{q}\right)$.

Theorem 2.6. Let $\ell \in \mathbb{F}_{q^{n}}$ be a q-linearized polynomial of linearized degree $r$ and in $t$-normalized form. Let $t \geq 1$ be a positive integer. Let $d$ be the $t$-scatter-degree of $\ell$. Let $C$ be a uniformizing constant for the extension $M / \mathbb{F}_{q^{n}}(s)$, and let $m \geq 1$ be such that $q^{m n}>C$. Then the following are equivalent:

(1) $\ell$ is $(q, n, m, t)$-scattered;

(2) for every $\gamma \in G_{m}^{\text {arith }}$ such that $\varphi_{m}(\gamma)$ is a Frobenius for $k_{m} / \mathbb{F}_{q^{m n}}$ and every $h \in G_{m}^{g e o m}$, the following condition holds:

$$
\operatorname{rk}(h \gamma-\mathrm{Id}) \geq d-1 \text {. }
$$

Moreover, $\ell$ is exceptional $t$-scattered if and only if there exists an $m \geq 1$ such that $q^{n m}>C$ and $\ell$ is $(q, n, m, t)$-scattered.

Proof. First, notice that $\ell(x) / x-s x^{q^{t}-1}$ is an irreducible polynomial in $\mathbb{F}_{q^{n m}}(s)[x]$. Let us set $L_{m}:=\mathbb{F}_{q^{n m}}(s)[x] /\left(\ell(x) / x-s x^{q^{t}-1}\right)$, which is simply $\mathbb{F}_{q^{n m}}(s, \alpha)$, where $\alpha$ is a non-zero root of $\ell-s x^{q^{t}}$.

$(1) \Longrightarrow(2)$ Let $\ell$ be $(q, n, m, t)$-scattered, and pick $\gamma \in G_{m}^{\text {arith }}$ with $\varphi_{m}(\gamma)$ the Frobenius automorphism for $k_{m} / \mathbb{F}_{q^{m n}}$ and any $h \in G_{m}^{\text {geom }}$. By Lemma 2.1, $h \gamma$ is a Frobenius for a finite, unramified place of degree 1 of $\mathbb{F}_{q^{m n}}(s)$ which we will denote by $P$. The polynomial $\ell / x-s_{0} x^{q^{t}-1}$, where $s_{0}$ is the value in $\mathbb{F}_{q}$ corresponding to $P$, has at most $q-1$ roots in $\mathbb{F}_{q^{m n}}$. Hence there are at most $q-1$ finite places of degree 1 of $L_{m}$ lying above $P$. Let $V$ be the $\mathbb{F}_{q}$-vector space of roots of $\ell-s x^{q^{t}}$, so that $V \backslash\{0\}$ is the set of roots of $\ell / x-s_{0} x^{q^{t}-1}$. By Corollary 2.4 it follows that the decomposition group $D(R \mid P) \subseteq G_{m}^{\text {arith }}$ has at most $q-1$ fixed points when acting on $V \backslash\{0\}$. Since $R \mid P$ is unramified, $D(R \mid P)$ 
is generated by $h \gamma$ and therefore $h \gamma$ has at most $q-1$ fixed points when acting on $V \backslash\{0\}$. But this is equivalent to asking that $h \gamma-\mathrm{Id}$ has rank at least $d-1$.

$(2) \Longrightarrow(1)$ Suppose by contradiction that $\ell$ is not $(q, n, m, t)$-scattered. Then there exists $s_{0} \in \mathbb{F}_{q^{n m}}$ such that $\ell-s_{0} x^{q^{t}}$ has at least $q^{2}-1$ non-zero roots in $\mathbb{F}_{q^{n m}}$ (notice in fact that if $\ell / x-s_{0} x^{q^{t}-1}$ has at least $q$ roots, then it has at least $q^{2}-1$ roots). Then there are at least $q^{2}-1$ finite places of degree 1 of $L_{m}$ lying above the place $P_{s_{0}}$ of $\mathbb{F}_{q^{n m}}(s)$ corresponding to $s_{0}$. Notice that these places are unramified in $L_{m}$ : if $P_{s_{0}}$ ramifies in $L_{m}$ then $\ell-s_{0} x^{q^{t}}$ has a multiple root. But this is impossible by assumption, because $t>0$ and $\ell$ has a non-zero linear term, and therefore the derivative of $\ell-s_{0} x^{q^{t}}$ is constant.

Hence all finite places of $L_{m}$ lying above $P_{s_{0}}$ are unramified, and at least $q^{2}-1$ of them have degree 1. Fix a place $R$ of $M_{m}$ lying above $P_{s_{0}}$. By Lemma 2.4, the decomposition group $D\left(R \mid P_{s_{0}}\right)$ has at least $q^{2}-1$ fixed points when acting on $V \backslash\{0\}$. Thus all elements of $D\left(R \mid P_{s_{0}}\right)$ enjoy the same property. Now notice that there is a surjective map $D\left(R \mid P_{s_{0}}\right) \rightarrow \operatorname{Gal}\left(k_{R} / k_{P_{s_{0}}}\right)$. Since $P_{s_{0}}$ has degree 1 then the residue field $k_{P_{s_{0}}}$ is $\mathbb{F}_{q^{m n}}$, and it is a standard fact that $k_{R}$ is an extension of $k_{m}$. Hence, there must exist $\gamma \in D\left(R \mid P_{s_{0}}\right)$ whose image via the aforementioned surjection is the Frobenius for $k_{m} / \mathbb{F}_{q^{m n}}$. It follows immediately that also $\varphi_{m}(\gamma)$ is the Frobenius for $k_{m} / \mathbb{F}_{q^{m n}}$. But then we have a contradiction with (2) because if $\gamma$ acts on $V \backslash\{0\}$ with at least $q^{2}-1$ fixed points, then $\operatorname{rk}(\gamma-\mathrm{Id}) \leq d-2$.

Finally, let us prove the last part of the statement. One direction is obvious. Thus, suppose $m \geq 1$ is such that $q^{n m}>C$ and $\ell$ is $(q, n, m, t)$-scattered. Then condition (2) holds. Now for all the infinitely many $y \in \mathbb{Z}_{\geq 1}$ that are coprime with $\left[k_{m}: \mathbb{F}_{q^{n m}}\right]$ we have that $G_{m}^{\text {arith }} \cong G_{m y}^{\text {arith }}$ and $G_{m}^{\text {geom }} \cong G_{m y}^{\text {geom }}$, and therefore (2) holds for infinitely many $y$ 's. It follows that $\ell$ is exceptional $t$-scattered.

Corollary 2.7. With the notation of Theorem 2.6, if $\ell$ is $(q, n, m, t)$-scattered then $G_{m}^{\text {geom }} \neq G_{m}^{\text {arith }}$.

Proof. If it was $G_{m}^{\text {geom }}=G_{m}^{\text {arith }}$, then $\varphi_{m}(\mathrm{Id})$ would be a Frobenius for $k_{m} / \mathbb{F}_{q^{n m}}$, and hence one should have, in particular, that $\operatorname{rk}(\mathrm{Id}-\mathrm{Id}) \geq d-1$, a clear contradiction.

\section{Finite transitive Linear Groups in odD Characteristic}

In [9], Hering classified all finite 2-transitive affine groups with abelian elementary socle (over finite fields). Looking at the stabilizer of a point, one can deduce the following classification theorem for finite transitive linear groups. Recall that if $q$ is a prime power 
and $a, b \geq 1$ we denote by $\Gamma L_{a}\left(\mathbb{F}_{q^{b}}\right)$ the general semilinear group, namely the semidirect product $\mathrm{GL}_{a}\left(\mathbb{F}_{q^{b}}\right) \rtimes \operatorname{Aut}_{\mathbb{F}_{q}}\left(\mathbb{F}_{q^{b}}\right) \cong \mathrm{GL}_{a}\left(\mathbb{F}_{q^{b}}\right) \rtimes C_{b}$.

Theorem 3.1 ([10, Theorem 69.7]). Let $p$ be an odd prime, $n \in \mathbb{Z}_{\geq 1}$ and $G$ be a subgroup of $\mathrm{GL}_{n}\left(\mathbb{F}_{p}\right)$ that acts transitively on $\mathbb{F}_{p}^{n} \backslash\{0\}$. Then $G$ satisfies one of the following.

(1) $\mathrm{SL}_{e}\left(\mathbb{F}_{p^{n / e}}\right) \leq G \leq \Gamma L_{e}\left(\mathbb{F}_{p^{n / e}}\right)$ for some e $\mid n$;

(2) $\operatorname{Sp}_{e}\left(\mathbb{F}_{p^{n / e}}\right) \leq G \leq \Gamma L_{e}\left(\mathbb{F}_{p^{n / e}}\right)$ for some even e $\mid n$;

(3) $n$ is even and $(p, n)$ belongs to a finite list of sporadic pairs.

When $d$ is prime, one can in turn deduce the following proposition.

Proposition 3.2. Let $q$ be an odd prime power, $d$ be an odd prime, and $G$ be a subgroup of $\mathrm{GL}_{d}\left(\mathbb{F}_{q}\right)$ that acts transitively on $\mathbb{F}_{q}^{d} \backslash\{0\}$. Then $G$ satisfies one of the following.

(1) $\mathrm{SL}_{d}\left(\mathbb{F}_{q}\right) \leq G \leq \mathrm{GL}_{d}\left(\mathbb{F}_{q}\right)$;

(2) $G \leq \Gamma L_{1}\left(\mathbb{F}_{q^{d}}\right)$.

Proof. Let $q=p^{a}$ where $p$ is a prime and $a \geq 1$. Since $\mathrm{GL}_{d}\left(\mathbb{F}_{q}\right) \leq \mathrm{GL}_{a d}\left(\mathbb{F}_{p}\right)$ and the group $G$ acts transitively on $\mathbb{F}_{p}^{a d} \backslash\{0\}$, we can apply Theorem 3.1 with $n=a d$ and deduce that one of the following holds:

(I) $\mathrm{SL}_{e}\left(\mathbb{F}_{p^{a d / e}}\right) \leq G \leq \Gamma L_{e}\left(\mathbb{F}_{p^{a d / e}}\right)$ for some $e \mid a d$;

(II) $\operatorname{Sp}_{e}\left(\mathbb{F}_{p^{a d} / e}\right) \leq G \leq \Gamma L_{e}\left(\mathbb{F}_{p^{a d} / e}\right)$ for some even $e \mid a d$;

(III) $a d$ is even and $(p, n)$ belongs to a finite list of sporadic pairs.

First, suppose that (I) holds for some $e \geq 3$. We claim that $e=d$, which implies immediately that $\mathrm{SL}_{d}\left(\mathbb{F}_{q}\right) \leq G \leq \mathrm{GL}_{d}\left(\mathbb{F}_{q}\right)$, since $\Gamma L_{d}\left(\mathbb{F}_{q}\right)=\mathrm{GL}_{d}\left(\mathbb{F}_{q}\right)$. Let us now split the proof into two cases.

Case 1: $d \mid e$. Since $\mathrm{SL}_{e}\left(\mathbb{F}_{p^{a d / e}}\right) \leq G$ and $G \leq \mathrm{GL}_{d}\left(\mathbb{F}_{q}\right)$ by hypothesis, we must have that $v_{p}\left(\left|\mathrm{SL}_{e}\left(\mathbb{F}_{p^{a d} / e}\right)\right|\right) \leq v_{p}\left(\left|\mathrm{GL}_{d}\left(\mathbb{F}_{q}\right)\right|\right)$, where $v_{p}$ is the usual $p$-adic valuation. However, a quick calculation shows that $v_{p}\left(\left|\mathrm{SL}_{e}\left(\mathbb{F}_{p^{a d} / e}\right)\right|\right)=\operatorname{ad}(e-1) / 2$, while $v_{p}\left(\left|\mathrm{GL}_{d}\left(\mathbb{F}_{q}\right)\right|\right)=$ $a d(d-1) / 2$, forcing $e=d$.

Case 2: $d \nmid e$. Also in this case we have $\mathrm{SL}_{e}\left(\mathbb{F}_{p^{a d / e}}\right) \leq G$ and $G \leq \mathrm{GL}_{d}\left(\mathbb{F}_{q}\right)$ by hypothesis. The condition $d \nmid e$ and the fact that $d$ is prime imply that $e \mid a$ and $(d, e)=1$. Let $\widetilde{p}:=p^{a / e}$. Let $r$ be a Zsigmondy prime for $\widetilde{p}^{d(e-1)}-1$, i.e. a prime that divides $\widetilde{p}^{d(e-1)}-1$ but does not divide $\widetilde{p}^{t}-1$ for any $1 \leq t<d(e-1)$, whose existence is guaranteed by Zsigmondy's Theorem (see [3, Theorem V]). It is immediate to check that $r|| \mathrm{SL}_{e}\left(\mathbb{F}_{p^{a d / e}}\right) \mid$ but $r \nmid\left|\mathrm{GL}_{d}\left(\mathbb{F}_{q}\right)\right|$, as et can't be a multiple of $d(e-1)$ for any $t \leq d$, yielding a contradiction. 
If (II) holds for some $e \geq 6$, one argues in a totally analogous way. For $e=4$, just notice that $\left|\operatorname{Sp}_{4}\left(\mathbb{F}_{p^{a d / 4}}\right)\right|=p^{a d}\left(p^{a d / 2}-1\right)\left(p^{a d}-1\right)$. Looking at a Zsigmondy prime or $p^{a d / 2}-1$, one sees immediately that $\left|\mathrm{Sp}_{4}\left(\mathbb{F}_{p^{a d / 4}}\right)\right|$ cannot divide $\left|\mathrm{GL}_{d}\left(\mathbb{F}_{q}\right)\right|$.

Next, assume (I) holds for $e=2$, which is the same as saying that (II) holds for $e=2$ (as $\mathrm{SL}_{2}\left(\mathbb{F}_{Q}\right)=\mathrm{Sp}_{2}\left(\mathbb{F}_{Q}\right)$ for any prime power $Q$ ). Since $d$ is odd, a must be even. Let $\widetilde{p}:=p^{a / 2}$. We claim that there is no embedding $\mathrm{SL}_{2}\left(\mathbb{F}_{\widetilde{p}^{d}}\right) \hookrightarrow \mathrm{GL}_{d}\left(\mathbb{F}_{\widetilde{p}^{2}}\right)$. Suppose by contradiction that there is one. Since both $p$ and $d$ are odd primes, $\mathrm{SL}_{2}\left(\mathbb{F}_{\widetilde{p}^{d}}\right)$ is perfect and $\left[\mathrm{GL}_{d}\left(\mathbb{F}_{\widetilde{p}^{2}}\right), \mathrm{GL}_{d}\left(\mathbb{F}_{\widetilde{p}^{2}}\right)\right]=\mathrm{SL}_{d}\left(\mathbb{F}_{\widetilde{p}^{2}}\right)$. Hence, if such embedding exists then there exists also an embedding $\iota: \mathrm{SL}_{2}\left(\mathbb{F}_{\widetilde{p}^{d}}\right) \hookrightarrow \mathrm{SL}_{d}\left(\mathbb{F}_{\widetilde{p}^{2}}\right)$. The group $\mathrm{SL}_{2}\left(\mathbb{F}_{\widetilde{p}^{d}}\right)$ contains an element $\sigma$ of order $\widetilde{p}^{d}+1$, which comes from a Singer cycle of $\mathrm{GL}_{2}\left(\mathbb{F}_{\widetilde{p}^{d}}\right)$. Now let $r$ be a Zsigmondy prime for $\widetilde{p}^{2 d}-1$. Clearly $r \mid \widetilde{p}^{d}+1$. Hence, the cyclic subgroup generated by $\iota(\sigma)$ contains an element $\tau$ of order $r$. Since $r$ is a Zsigmondy prime for $\widetilde{p}^{2 d}-1$, one can check that the cyclic group $H$ generated by $\tau$ acts irreducibly on $\mathbb{F}_{\widetilde{p}^{2}}^{d}$. Hence, by Schur's Lemma and the fact that finite division rings are fields, the centralizer $C(H)$ of $H$ in $\mathrm{GL}_{d}\left(\mathbb{F}_{\widetilde{p}^{2}}\right)$ is the multiplicative group of a finite field of characteristic $p$. Since $r|| C(H) \mid$, then it must be $|C(H)|=\widetilde{p}^{2 d}-1$; in other words $C(H)$ arises from a Singer cycle. Thus its intersection with $\mathrm{SL}_{d}\left(\mathbb{F}_{\widetilde{p}^{2}}\right)$ has order $\left(\widetilde{p}^{2 d}-1\right) /\left(\widetilde{p}^{2}-1\right)$, which is not divisible by $\widetilde{p}^{d}+1$. This gives a contradiction, so there is no such embedding and (I) cannot hold for $e=2$.

Finally, the only transitive sporadic group that can appear as a subgroup of $\mathrm{GL}_{a d}\left(\mathbb{F}_{p}\right)$ with $p, d$ odd primes is $\mathrm{SL}_{2}\left(\mathbb{F}_{13}\right)$, which is contained in $\mathrm{GL}_{6}\left(\mathbb{F}_{3}\right)$. This necessarily comes from the setting in which $q=9$ and $d=3$. But one can check with Magma [4] that $\mathrm{SL}_{2}\left(\mathbb{F}_{13}\right)$ does not embed in $\mathrm{GL}_{3}\left(\mathbb{F}_{9}\right)$, concluding the proof of the proposition.

\section{Exceptional SCATtered POlynomials of PRime DEGREe}

The goal of this section is to prove Theorem 1.2. The proof relies on two fundamental ingredients: the Galois theoretical characterization of exceptional scattered polynomials given by Theorem 2.6 and the classification of finite transitive groups given by Proposition 3.2 .

Let us recall the setup, which is the one explained in Section 2. Let $\ell \in \mathbb{F}_{q^{n}}[x]$ be a $q$-linearized, exceptional $t$-normalized $t$-scattered polynomial of linearized degree $r$, and let $t>0$. Let $M$ be the splitting field of $\ell-s x^{q^{t}}$ over $\mathbb{F}_{q^{n}}(s)$; for every $m \geq 1$ let $M:=M \cdot \mathbb{F}_{q^{n m}}(s), k_{m}:=\overline{\mathbb{F}}_{q} \cap M_{m}$ and let $G_{m}^{\text {arith }}:=\operatorname{Gal}\left(M_{m} / \mathbb{F}_{q^{n m}}(s)\right)$ and $G_{m}^{\text {geom }}:=$ $\operatorname{Gal}\left(M_{m} / k_{m} \cdot \mathbb{F}_{q^{n}}(s)\right)$. Finally, we let $d:=\max \{r, t\}$ and $V$ be the set of roots of $\ell-s x^{q^{t}}$, which is an $\mathbb{F}_{q}$-vector space. 
We begin by showing that in the setting of Theorem 1.2, there is a very restrictive condition on $G_{m}^{\text {arith }}$ and $G_{m}^{\text {geom }}$.

Lemma 4.1. Let $t \geq 2, n$ be an integer, $q$ be a prime power and let $\ell \in \mathbb{F}_{q^{n}}[x]$ be a $q$-linearized, exceptional $t$-normalized $t$-scattered polynomial of linearized degree $r$. Let $M, G^{\text {arith }}, G^{\text {geom }}$ be defined as above. Assume that $d:=\max \{r, t\}$ is an odd prime. Let $C$ be the uniformizing constant for the extension $M / \mathbb{F}_{q^{n}}(s)$, and suppose $m \geq 1$ is such that $q^{n m}>C$ and $\ell$ is $(q, n, m, t)$-scattered. Then $\left|G_{m}^{\text {geom }}\right|=q^{d}-1$ and $G_{m}^{\text {arith }} \cong \Gamma L_{1}\left(q^{d}\right)$.

Proof. Before starting the proof, let us recall the following elementary facts, that will be helpful later on:

$$
\left|\mathrm{GL}_{d}\left(\mathbb{F}_{q}\right)\right|=\prod_{i=0}^{d-1}\left(q^{d}-q^{i}\right), \quad \mathrm{GL}_{d}\left(\mathbb{F}_{q}\right) \cong \mathrm{SL}_{d}\left(\mathbb{F}_{q}\right) \rtimes \mathbb{F}_{q}^{*} .
$$

so that in particular $(q-1)\left|\mathrm{SL}_{d}\left(\mathbb{F}_{q}\right)\right|=\left|\mathrm{GL}_{d}\left(\mathbb{F}_{q}\right)\right|$.

Now, since $\left(\ell-s x^{q^{t}}\right) / x$ is absolutely irreducible, $G_{m}^{\text {geom }}$ acts transitively on $V \backslash\{0\}$. Thus by Proposition 3.2 we only have two possibilities for $G_{m}^{\text {geom }}$ : either $\operatorname{SL}_{d}\left(\mathbb{F}_{q}\right) \leq$ $G_{m}^{\text {geom }} \leq \mathrm{GL}_{d}\left(\mathbb{F}_{q}\right)$ or $G_{m}^{\text {geom }} \leq \Gamma L_{1}\left(q^{d}\right)$. As a first step of the proof, we will show that $\mathrm{SL}_{d}\left(\mathbb{F}_{q}\right) \leq G_{m}^{\text {geom }} \leq \mathrm{GL}_{d}\left(\mathbb{F}_{q}\right)$ leads to a contradiction. In fact, suppose that such inclusion holds, and let $\gamma \in G^{\text {arith }}$ be a Frobenius for the field of constants $k_{m}$ of $M_{m}$. Any element of the coset $G_{m}^{\text {geom }} \gamma$ is a Frobenius for $k_{m}$. Hence, up to multiplying on the left by an element of $\mathrm{SL}_{d}\left(\mathbb{F}_{q}\right)$, we can assume by (1) that $\gamma$ has the form $\left(\lambda_{i, j}\right)_{i, j=1, \ldots, d}$ where $\lambda_{1,1}=\lambda \in \mathbb{F}_{q}^{*}, \lambda_{i, i}=1$ for $i>1$ and $\lambda_{i, j}=0$ otherwise. This can be seen by noticing that a splitting of the short exact sequence $\mathrm{SL}_{d}\left(\mathbb{F}_{q}\right) \hookrightarrow \mathrm{GL}_{d}\left(\mathbb{F}_{q}\right) \rightarrow \mathbb{F}_{q}^{*}$ is the map $\mathbb{F}_{q}^{*} \hookrightarrow \mathrm{GL}_{d}\left(\mathbb{F}_{q}\right)$ that sends each $\lambda \in \mathbb{F}_{q}^{*}$ to the matrix $\left(\lambda_{i, j}\right)_{i, j}$ described above. But then, $\operatorname{rk}(\gamma-\mathrm{Id}) \leq 1<d-1$, contradicting Theorem 2.6.

Hence, we must have $G_{m}^{\text {geom }} \leq \Gamma L_{1}\left(q^{d}\right)$. Since $G_{m}^{\text {geom }}$ acts transitively on $V \backslash\{0\}$, which has cardinality $q^{d}-1$, it follows by the Orbit-Stabilizer Theorem that $\left(q^{d}-1\right)|| G_{m}^{\text {geom }} \mid$. On the other hand, $\left|\Gamma L\left(1, q^{d}\right)\right|=d\left(q^{d}-1\right)$ and hence $\left|G_{m}^{\text {geom }}\right| \in\left\{q^{d}-1, d\left(q^{d}-1\right)\right\}$ because $d$ is prime. Now we claim that we must have $G_{m}^{\text {arith }} \leq \Gamma L\left(1, q^{d}\right)$. Notice that this ends the proof, because $G_{m}^{\text {geom }} \unlhd G_{m}^{\text {arith }}$, and hence it can only be $G_{m}^{\text {arith }}=G_{m}^{\text {geom }}$ or $G_{m}^{\text {arith }}=\Gamma L\left(1, q^{d}\right)$; however $G_{m}^{\text {arith }}=G_{m}^{\text {geom }}$ cannot occur by Corollary 2.7.

To prove that $G_{m}^{\text {arith }} \leq \Gamma L\left(1, q^{d}\right)$, we proceed by contradiction. By Proposition 3.2, the only other possibility is that $\mathrm{SL}_{d}\left(\mathbb{F}_{q}\right) \unlhd G_{m}^{\text {arith }} \leq \mathrm{GL}_{d}\left(\mathbb{F}_{q}\right)$. If this holds, since $G_{m}^{\text {geom }} \unlhd$ $G_{m}^{\text {arith }}$ we must have that $G_{m}^{\text {geom }} \cap \mathrm{SL}_{d}\left(\mathbb{F}_{q}\right) \unlhd \mathrm{SL}_{d}\left(\mathbb{F}_{q}\right)$. If $G_{m}^{\text {geom }} \cap \mathrm{SL}_{d}\left(\mathbb{F}_{q}\right)=\mathrm{SL}_{d}\left(\mathbb{F}_{q}\right)$ then $\left|\mathrm{SL}_{d}\left(\mathbb{F}_{q}\right)\right|$ must divide either $q^{d}-1$ or $d\left(q^{d}-1\right)$, and it is immediate to see that this is 
impossible because of (1). On the other hand, since $d \geq 3$ and $\mathrm{PSL}_{d}\left(\mathbb{F}_{q}\right)$ is simple, the only non-trivial normal subgroups of $\mathrm{SL}_{d}\left(\mathbb{F}_{q}\right)$ are those contained in the intersection of $\mathrm{SL}_{d}\left(\mathbb{F}_{q}\right)$ with the center of $\mathrm{GL}_{d}\left(\mathbb{F}_{q}\right)$ (see [6]). Thus if $G_{m}^{\text {geom }} \cap \mathrm{SL}_{d}\left(\mathbb{F}_{q}\right) \neq \mathrm{SL}_{d}\left(\mathbb{F}_{q}\right)$ then we have that, in particular, $\left|\mathrm{SL}_{d}\left(\mathbb{F}_{q}\right) \cap G_{m}^{\text {geom }}\right| \leq q-1$. Hence

$$
\left|\mathrm{GL}_{d}\left(\mathbb{F}_{q}\right)\right| \geq\left|G_{m}^{\text {geom }} \cdot \mathrm{SL}_{d}\left(\mathbb{F}_{q}\right)\right|=\frac{\left|G_{m}^{\text {geom }}\right|\left|\mathrm{SL}_{d}\left(\mathbb{F}_{q}\right)\right|}{\left|G_{m}^{\text {geom }} \cap \mathrm{SL}_{d}\left(\mathbb{F}_{q}\right)\right|} \geq \frac{\left(q^{d}-1\right)\left|\mathrm{GL}_{d}\left(\mathbb{F}_{q}\right)\right|}{(q-1)^{2}}
$$

which is a contradiction because $d \geq 3$ and therefore $\left|\mathrm{GL}_{d}\left(\mathbb{F}_{q}\right)\right|<\frac{\left(q^{d}-1\right)\left|\mathrm{GL}_{d}\left(\mathbb{F}_{q}\right)\right|}{(q-1)^{2}}$.

Proof of Theorem 1.2. Let $C$ be the uniformizing constant for $M / \mathbb{F}_{q^{n}}(s)$. Since $\ell$ is exceptional $t$-scattered, there exists $m \geq 1$ such that $q^{m n}>C$ and $\ell$ is $(q, n, m, t)$ scattered. By Lemma 4.1 it follows immediately that the constant field of $M_{m}$ is $k_{m}=$ $\mathbb{F}_{q^{d n m}}$ and that the splitting field $M_{m}$ of $\ell-s x^{q^{t}}$ over $\mathbb{F}_{q^{d n m}}(s)$ is simply $\mathbb{F}_{q^{d n m}}(s)[x] /(\ell / x-$ $s x^{q^{t}-1}$ ) because it contains one root and it has the correct degree. Notice that $M_{m}$ is isomorphic to the rational function field over $\mathbb{F}_{q^{d n m}}$, and hence its places of degree 1 are in 1-1 correspondence with the $\mathbb{F}_{q^{d n m}}$-rational points of the projective line.

From now on, we will denote by $P_{0}$ and $P_{\infty}$ the places of $\mathbb{F}_{q^{d n m}}(s)$ corresponding to 0 and to $\infty$, respectively. Similarly, we will denote by $Q_{0}$ and $Q_{\infty}$ the places of $M_{m}$ corresponding to 0 and to $\infty$, respectively.

Now we have to distinguish two cases, namely $t<r$ and $t>r$.

If $t<r$, then consider the place $P_{\infty}$. The only two places of $M_{m}$ that lie above $P_{\infty}$, are $Q_{0}$ and $Q_{\infty}$. It is immediate to check that the ramification index of $Q_{0} / P_{\infty}$ is $\left(q^{r}-1\right)-\left(q^{t}-1\right)=q^{t}\left(q^{r-t}-1\right)$. Since $t>0$, it follows that this ramification index is divisible by $q$, and this is impossible since $M_{m} / \mathbb{F}_{q^{d n m}}(s)$ is a Galois extension of degree $q^{d}-1$, which is coprime with $q$.

If $t>r$, we look at $P_{0}$ : the place $Q_{\infty}$ lies above it and has ramification index $q^{t}-q^{r}$. If $r>0$, this is divisible by $q$ and we are led to a contradiction by the same argument we used in the case $t<r$. Therefore it must be $r=0$, i.e. $\ell=x$.

\section{REFERENCES}

[1] Daniele Bartoli and Maria Montanucci. Towards the full classification of exceptional scattered polynomials. https://arxiv.org/abs/1905.11390, 2019.

[2] Daniele Bartoli and Yue Zhou. Exceptional scattered polynomials. J. Algebra, 509:507-534, 2018. 
[3] Geo. D. Birkhoff and H. S. Vandiver. On the integral divisors of $a^{n}-b^{n}$. Ann. of Math. (2), 5(4):173-180, 1904.

[4] Wieb Bosma, John Cannon, and Catherine Playoust. The Magma algebra system. I. The user language. J. Symbolic Comput., 24(3-4):235-265, 1997. Computational algebra and number theory (London, 1993).

[5] Bence Csajbók, Giuseppe Marino, Olga Polverino, and Ferdinando Zullo. Maximum scattered linear sets and MRD-codes. J. Algebraic Combin., 46(3-4):517-531, 2017.

[6] Leonard Eugene Dickson. Theory of linear groups in an arbitrary field. Trans. Amer. Math. Soc., 2(4):363-394, 1901.

[7] Andrea Ferraguti and Giacomo Micheli. Full classification of permutation rational functions and complete rational functions of degree three over finite fields. Des. Codes Cryptogr. (to appear), 2020.

[8] Robert M. Guralnick, Thomas J. Tucker, and Michael E. Zieve. Exceptional covers and bijections on rational points. Int. Math. Res. Not. IMRN, (1):Art. ID rnm004, 20, 2007.

[9] Christoph Hering. Transitive linear groups and linear groups which contain irreducible subgroups of prime order. II. J. Algebra, 93(1):151-164, 1985.

[10] Norman L. Johnson, Vikram Jha, and Mauro Biliotti. Handbook of finite translation planes, volume 289 of Pure and Applied Mathematics (Boca Raton). Chapman \& Hall/CRC, Boca Raton, FL, 2007.

[11] Michiel Kosters. A short proof of the Chebotarev density theorem for function fields. Math. Commun., 22(2):227-233, 2017.

[12] Giacomo Micheli. Constructions of locally recoverable codes which are optimal. IEEE Transactions on Information Theory, 2019.

[13] Giacomo Micheli. On the selection of polynomials for the DLP quasi-polynomial time algorithm for finite fields of small characteristic. SIAM J. Appl. Algebra Geom., 3(2):256-265, 2019.

[14] Sara Rottey and John Sheekey. A geometric characterisation of Desarguesian spreads. J. Algebraic Combin., 46(2):455-474, 2017.

[15] John Sheekey. A new family of linear maximum rank distance codes. Adv. Math. Commun., 10(3):475-488, 2016.

[16] John Sheekey. MRD codes: Constructions and connections. https://arxiv.org/abs/1904.05813, 2019. 
[17] Henning Stichtenoth. Algebraic function fields and codes, volume 254 of Graduate Texts in Mathematics. Springer-Verlag, Berlin, second edition, 2009.

Instituto de Ciencias Matemáticas, Calle Nicolás Cabrera 13, 28049 Madrid, Spain, E-mail address: and.ferraguti@gmail.com

University of South Florida, 4202 E Fowler Ave, 33620 Tampa, US.

E-mail address: gmicheli@usf .edu 